\title{
Application-Independent Localization Based on 3D Simulation Technology
}

\author{
Bjoern Sondermann, Markus Emde, and Juergen Rossmann
}

\begin{abstract}
Mobile robots are recognized as being essential for the examination of hazardous and dangerous places like disaster areas, underground mining and extraterrestrial environments. In this paper we introduce an application-independent approach for self-localization of mobile robots. The idea was implemented and optimized for forestry environments. A generalization of the concept led to a highly modular framework that is adaptable to a multitude of new domains. The framework is based on 3d-simulation technology and benefits from latest developments in this domain, like hybrid testbeds. This testbed approach allows for the integration of real and simulated sensors in virtual and real testbeds for a smooth transition between simulation and real world tests. Introducing virtual sensors, algorithmic results can be treated as ordinary sensor information and are therefore seamlessly addressed by the sensor framework.
\end{abstract}

Index Terms-Self-localization, virtual testbeds, sensor simulation, simulation frameworks.

\section{INTRODUCTION}

Our previous work focused on the development of new methods for highly accurate self-localization and navigation of work machines in the forest [1]. Fusing various sensors and implementing localization algorithms using Sequential Monte Carlo methods (SMC) led to a robust localization unit appropriate for unstructured environments. Mounted on a forest machine, this system allowed for the detection and localization of trees in the surrounding area, as well as the self-localization of the vehicle and its well-directed navigation.

We generalized and extended the underlying concept to an application-independent localization framework allowing to transfer the approach from forestry to other domains. The resulting localization framework is able to handle different classes of landmarks and sensors. The initial implementation was restricted to use laser scanners for landmark detection. With the re-adaptation of the general implementation to the forestry scenario, we are able to use any sensor to perceive the environment.

The development of the data processing algorithms has

Manuscript received March 24, 2015; revised July 27, 2015. Parts of this work were developed in the context of the research projects Virtual Forest, SELOK and FastMap. Virtual Forest is co-financed by the European Union and the federal state of North Rhine-Westphalia, European Regional Development Fund (ERDF). Europe - Investing in our future. SELOK/FastMap were supported by German Aerospace Center (DLR) with funds of the German Federal Ministry of Economics and Technology (BMWi), support code 50 RA 0911 (SELOK) and 50 RA 1034 (FastMap).

The authors are with the Institute for Man-Machine Interaction at RWTH Aachen University, Aachen, Germany (e-mail: sondermann@mmi.rwth-aachen.de, emde@mmi.rwth-aachen.de, rossmann@mmi.rwth-aachen.de). been accelerated using simulation software. Here, a modular sensor framework has been used to interact with real and simulated sensors. Furthermore, this sensor framework provides mechanisms to use algorithmic results as ordinary sensor data, called virtual sensors. The integration of the developed components in our testbed concept allows for evaluating algorithms and sensors in virtual, real and hybrid testbeds. Virtual testbeds are a cost-efficient alternative e.g. for the preparation of planetary exploration missions, especially if setting up real mockups for testing and verifying is too expensive. Our virtual testbed implements a $3 \mathrm{~d}$ geometric and functional simulation model incorporating the mobile robot, sensors and the environment. In addition the simulation system allows for testing and verifying the localization and control algorithms. Furthermore, it provides the possibility to rerun test series with slightly different parameters to find the best parameter sets.

\section{LOCALIZATION STRATEGY}

Exploration of unknown planetary surfaces with the help of mobile robots is essential for the success of current and future space missions, as robots can operate without danger in inhabitable places. Due to the long distances to those planets, instant and gapless communication is not available. Thus for continuous operation, these mobile robots need the ability for perceiving their environment and estimating their current position. Starting with a self-localization approach optimized and validated for the forest domain, we introduce a generalized and modular concept applicable in different environments.

Previous localization strategy for terrestrial unstructured environments: As described in [1] a forest machine has been equipped with several sensors to perceive its environment and to be able to determine its position with higher accuracy than by only using GPS receivers. The foundation of this approach is the use of trees as natural landmarks in the forest, as they are easily detectable and sufficiently available. We consider that a global tree map is given by a preliminary step [2], using remote sensing data like aerial images from satellites or planes. Fig. 1 illustrates the approach.

The localization algorithm is based on a particle filter algorithm as described in [3]. In [4] the calculation steps are described in detail, as well as information on parameterization of the algorithm.

Generalization: The aforementioned system has been tested, evaluated and optimized in forest stands over the last few years. Abstracting the components of the localization approach and generalizing the communication among them led to a new modular and flexible concept as shown in Fig. 2. 
The self-localization approach for forest environments has been broken down into modules, which have been implemented context-independent, so that they can be reused in different application areas. Furthermore, modules of the same kind, e.g. landmark detectors, can be substituted without effecting the other modules of the processing chain.

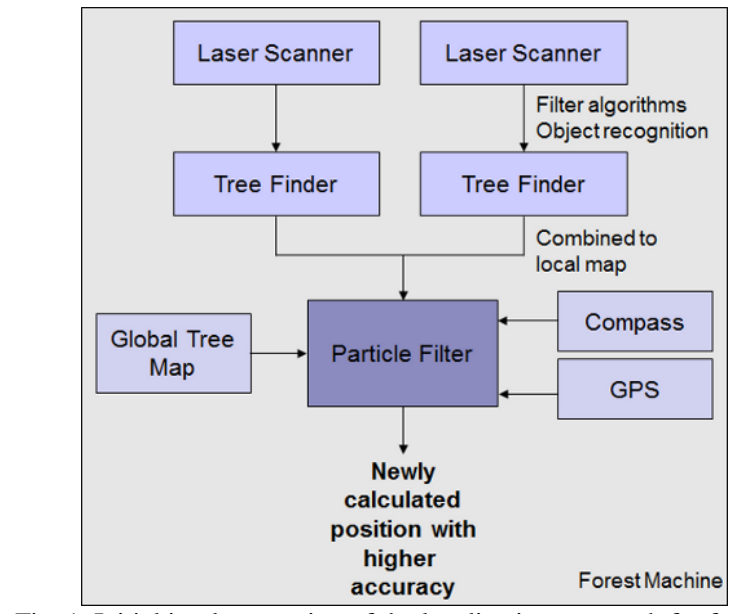

Fig. 1. Initial implementation of the localization approach for forestry environments.

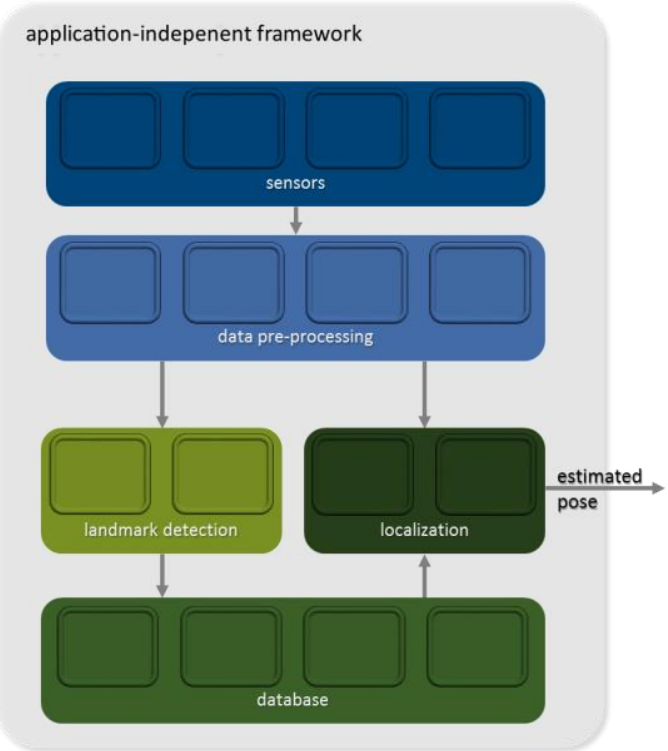

Fig. 2. Generalized localization concept.

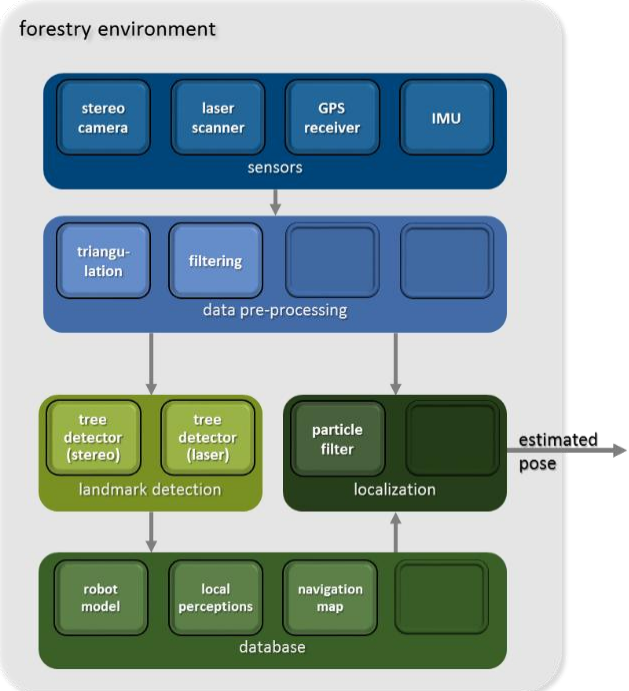

Fig. 3. Re-adaptation of the generalized concept to forestry environments.
The re-adaptation of the generalized self-localization approach to forestry environments is shown in Fig. 3. The general concept can now be used for further localization tasks, such as extraterrestrial exploration missions, or in environments, where GPS is not accurate enough or simply not available.

Adoption to planetary explorations: By the abstraction and modular reassembly of the localization unit, attaching new sensors is a straight forward task. Furthermore the modular landmark detector unit allows for many different detection instances as tree detectors in forest environments or for example rock detectors in extraterrestrial environments. Each detector instance defines the required sensors and for each used sensor class a particular landmark detector extracts a set of landmarks from the sensor data and provides it to the localizer. As we are using a general landmark definition, it is irrelevant which sensor was used for detection. In extraterrestrial environments many different types of landmarks are usable, for example rocks, craters, hills and so on. Therefore, the localization module has to evaluate the landmarks semantic information, as well as their position to match them correctly to the navigation map, wherein the landmarks are divided in the same semantic classes. Fig. 4 illustrates the adoption of the general concept to planetary explorations using rocks as landmarks for localization.

Landmark detection on stereo image data: The detection of landmarks using stereo cameras has been subject of many publications like in [5] and [6]. In most of them, the object detection is carried out on the input images directly. A better approach to solve the problem of landmark detection is to first compute a dense depth map from the stereo input images using one of the methods analyzed in [7]. We use an implementation of the semi global block matching method as introduced in [8].

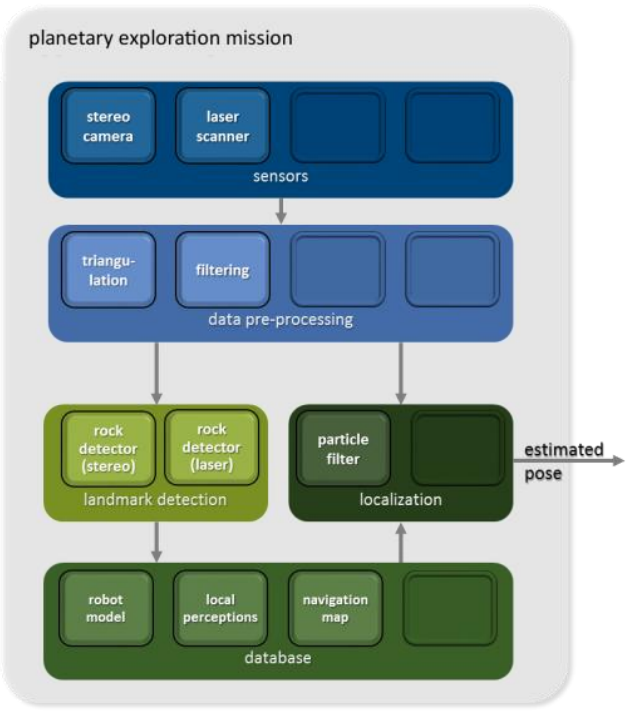

Fig. 4. Adaptation to planetary exploration missions.

Our tree detection algorithm selects all regions with little variations in their depth values and evaluates their main axis by evaluating the principal component analysis (PCA) of the area. If the ratio between the second and first axis of the PCA is under a given threshold, the region is marked as a possible tree. Additional parameters like declination against the ground and diameter are estimated as well. Thus, it is possible 
to decline the detection of trees that do not fulfill certain user defined requirements.

More challenging landmarks are rocks. A detector has to consider more aspects than the depth values from laser scanner or stereo camera as the geometry of rocks is arbitrary. Our approach is based on the idea of [9]. Instead of searching for rocks in the incoming stereo depth map or point cloud directly, some preprocessing is done to transform the distance measures of the stereo data into a height map according to a floor plane (see Fig. 5). With this transformation we can use fast image processing techniques for finding connected structures, as described in [10]. According to a compactness measure, evaluated by the ratio between circumference and area in the image space, possible compact rock landmarks can be distinguished from longish elevations of the ground.

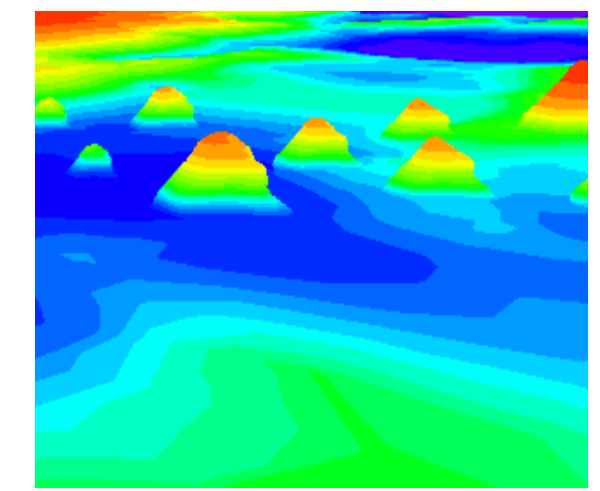

Fig. 5. Segmented height map with 21 height classes.

\section{VIRTUAL AND HyBRID TESTBEDS}

As already mentioned, the implemented approach had to be re-engineered to meet the requirements of new domains. In addition, connections among the implemented components should be flexible and modular. To meet all these requirements the underlying concepts to connect components and to communicate among them have been revised. The design allows easy setups of virtual and hybrid testbeds [11] using a generic communication concept for the interaction of all components. The developed sensor framework integrates four different types of sensors into the system and provides a smooth transition between simulation and real world tests [12]. Besides real, simulated and playback sensors, virtual sensors represent a class of sensors in this framework and are used to feed algorithmic results or recorded data into the network of connected components. As the data ports use an abstract base class for the envisioned types of data, recorded data or algorithmic results are treated as conventional sensor data. Regarding the introduced localization approach in this paper the result is provided as a virtual GPS-receiver, setting the absolute position information on the output of this component, but its position estimation is based on optical sensor data. The testbeds allow a focused view on every component of the system to analyze and optimize their behavior.

Testing: The ability of parallel testing in real, simulated and hybrid environments and the smooth transition between simulation and real world measurements is the main aspect of our testbed approach. Modules can be developed in a pure virtual environment and after an iterative calibration process with real components, they are validated and can be used in real environments as well. Therefore, the implemented modules are developed and tested context-independent, resulting in all-purpose modules. In general the development process is divided in the following iterative steps:

- Using a conceptual implementation in the testbed allows estimating the basic plausibility. All necessary sensor data is provided as ideal simulated sensor output for the developed algorithms.

- Error models are added to the simulated sensor data to measure the robustness of the developed algorithms, resulting in first requirements for the applicable hardware in the target system.

- The input data of the simulated components is continuously transformed from ideal to realistic by adding further error models. The identification of errors in the algorithms is always possible by moving individual components independently of the rest in the degree of their realism.

- Finally, all components of the simulation are replaced by their real counterparts for testing the algorithms on the target system.

Furthermore, the presented concept allows simulation-based engineering combining development strategies like hardware-in-the-loop, co-design and concurrent engineering. Starting in a virtual environment, targeted systems are implemented by replacing the simulated components successively by their physical counterparts leading to a final setup with real hardware. Finally, the simulation system itself is used to command the localization unit and to visualize the raw sensor and processed data in the virtual environment. This implies that for every physical component a simulated counterpart exists as illustrated in Fig. 6.

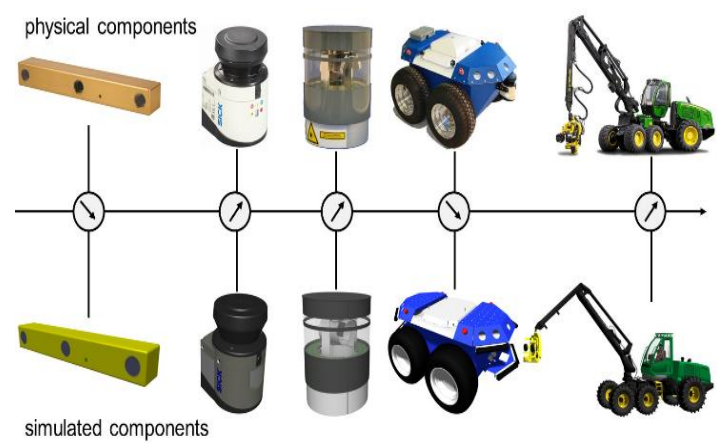

Fig. 6. Simulated components and their physical counterparts.

\section{RESUlts}

The results of the localization components have been evaluated in simulation during development and implementation using virtual testbeds. The localization results in the virtual testbed were compared to ground truth known from the simulation system. We tested our algorithms in both scenarios, forest and planetary exploration. In the forest the localization accuracy lies within a mean translational error of 0.1 meters. In the scenario planetary exploration the mean translational error lies within the dimension of 0.2 meters.

The implemented algorithms were also tested in physical 
testbeds. Here, the ground-truth is more difficult to achieve. In the forest, a surveyor team was hired to measure the position of the robot from calibrated measuring points. Therefore, the robot was equipped with two well visible markers. The tree positions were also manually measured by the surveyors and recorded in the navigation map. During the test runs the robot was stopped in frequent intervals and the surveyors measured the accurate position as a reference for our localization analysis. The mean accuracy in translation has been measured under 0.1 meters.

The real world planetary exploration tests have been carried out in a stone quarry. To verify the results of the localization as exact as possible, the rock landmarks were stored in the navigation map manually. The terrain was pictured and geo-tagged by a remote controlled aircraft and in addition $3 \mathrm{~d}$ laser scans were made to use the data inside the simulation system for further analyses. The start and end position of the robot was determined by measuring the distance of a marker on the robot from two measuring points. The robots movement was also recorded with a camera for identifying approximate deviations between localization and actual movement. The localization error at start and end position averages out at 0.25 meters.

\section{FUTURE WORK}

Simulated sensors with corresponding error models and real ones are already integrated into our system. The sensor data can be visualized as comprehensive metaphors allowing easy understanding of complex correlations during development processes. Furthermore the generated sensor data can be logged and played back allowing to rerun test series with slightly different parameters for optimization purposes.

Currently, we are working on adaptations to domains, where GNSS-services are not available like indoor applications or underground mining facilities. Our next goal is to use the presented localization concept in the domain of automated guided vehicle systems (AGV) for warehouse logistics. This adaptation can be achieved with little effort. Here, artificial markers like QR-tags are used as landmarks. These tags can also carry additional information, so that the reliability and accuracy of the localization can be increased. We are planning to use an omnidirectional camera as primary sensor, because its field-of-view covers the whole surrounding area and allows a maximum number of landmarks. A first prototype in a virtual testbed shows promising results.

\section{REFERENCES}

[1] J. Rossmann, M. Schluse, C. Schlette, A. Buecken, P. Krahwinkler, and M. Emde, "Realization of a highly accurate mobile robot system for multi purpose precision forestry applications," presented at 14th International Conference on Advanced Robotics (ICAR), Munich, Germany, 2009.

[2] J. Rossmann, G. Jochmann, and F. Bluemel, "Semantic navigation maps for mobile robot localization on planetary surfaces," presented at 12th Symposium on Advanced Space Technologies in Robotics and Automation (ASTRA), Noordwijk, The Netherlands, 2013.

[3] S. Thrun, W. Burgard, and D. Fox, Probabilistic Robotics, MIT Press, USA, 2005.

[4] J. Rossmann, C. Schlette, M. Emde, and B. Sondermann, "Advanced self-localization and navigation for mobile robots in extraterrestrial environments," Computer Technology and Application, vol. 2, no. 5, pp. 344-353, 2011.

[5] M. N. Dailey and M. Parnichkun, "Landmark-based simultaneous localization and mapping with stereo vision," presented at Asian Conference on Industrial Automation and Robotics, Bangkok, Thailand, 2005.

[6] S. C. Hirtle, "Landmarks for navigation in human and robots," Robotics and Cognitive Approaches to Spatial Mapping, Springer, pp. 203-214, 2008.

[7] D. Scharstein, R. Szeliski, and R. Zabih, "A taxonomy and evaluation of dense two-frame stereo correspondence algorithms," IEEE Workshop on Stereo and Multi-Baseline Vision, vol. 47, no. 1, pp. 131-140, 2001.

[8] H. Hirschmüller, "Stereo processing by semiglobal matching and mutual information," IEEE Transactions on Pattern Analysis and Machine Intelligence, vol. 30, no. 2, pp. 328-41, 2008.

[9] V. Gor, R. Castano, and R. Manduchi, "Autonomous rock detection for Mars terrain," American Institute of Aeronautics and Astronautics Space (AIAA) 2001, Conference \& Exposition, 2001.

[10] M. Emde, B. Sondermann, and J. Roßmann, "A self-contained localization unit for terrestrial applications and its use in space environments," presented at the 12th International Symposium on Artificial Intelligence, Robotics and Automation in Space (i-SAIRAS) 2014, Montreal, Canada, 2014

[11] B. Sondermann, M. Emde, M. Rast, and J. Rossmann, "Simulation-based engineering with hybrid testbeds," presented at European Modelling Symposium 2013, pp. 471-476, 2013.

[12] M. Emde, J. Rossmann, B. Sondermann, and N. Hempe, "Advanced sensor simulation In virtual testbeds: A cost-efficient way to develop and verify space applications," presented at American Institute of Aeronautics and Astronautics in Space (AIAA) 2011, Conference \& Exposition, Long Beach, USA, 2011

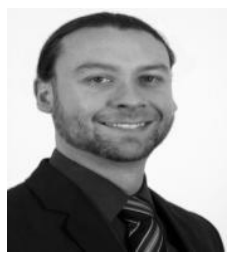

Bjoern Sondermann studied computer science at the University of Bonn, Germany. Since 2009, he works as a researcher at the Institute for Man-Machine Interaction (MMI) in Aachen. His research interests include computer vision, environment perception and localization of mobile robots.

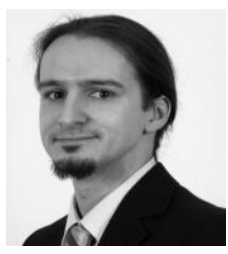

Markus Emde studied computer science at the University of Bremen, Germany. Since 2006 he works as a researcher at the Institute for Man-Machine Interaction (MMI) in Aachen. His research interests include space, industrial robotics. He focused on sensor integration, sensor simulation and data processing techniques.

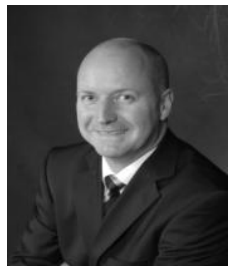

Juergen Rossmann studied electrical engineering a the Universities of Dortmund and Bochum, Germany. After his studies, he worked as a researcher and a team leader at the Institute of Robotics Research (IRF) in Dortmund. He received his doctorate in 1993 and was appointed as a visiting assistant professor for robotics and computer graphics at the University of Southern California in 1998. He received his habilitation degree in 2002 from the University of Dortmund and was the managing director of EFR-Systems GmbH in Dortmund from 2005 to 2006.

Since 2006, he has been the director of the Institute for Man-Machine Interaction and a full professor at the RWTH Aachen University in Aachen, Germany. His research interests are projective virtual reality, multi-agent control and supervision, multi-sensor integration, system simulation and optimisation techniques, computer vision, real-time visualisation and man-machine interaction. 